\title{
LC-MS/MS Analysis Elucidates a Daily Rhythm in Orexin A Concentration in the Rat Vitreous Body
}

\author{
Lukasz Chrobok $^{1, *(\mathbb{D})}$, Sylwia Bajkacz ${ }^{2,3, *(\mathbb{D}}$, Jasmin Daniela Klich ${ }^{1}\left[\right.$ and Marian Henryk Lewandowski $^{1}(\mathbb{D})$ \\ 1 Department of Neurophysiology and Chronobiology, Institute of Zoology and Biomedical Research, \\ Jagiellonian University in Krakow, Gronostajowa 9 Street, 30-387 Krakow, Poland; \\ klich.jasmina@gmail.com (J.D.K.); marian.lewandowski@uj.edu.pl (M.H.L.) \\ 2 Department of Inorganic Chemistry, Analytical Chemistry and Electrochemistry, Faculty of Chemistry, \\ Silesian University of Technology, Krzywoustego 6 Street, 44-100 Gliwice, Poland \\ 3 The Biotechnology Centre, Silesian University of Technology, Krzywoustego 8 Street, 44-100 Gliwice, Poland \\ * Correspondence: lukasz.chrobok@uj.edu.pl (L.C.); sylwia.bajkacz@polsl.pl (S.B.); Tel.: +48-(12)-6645044 (L.C.); \\ $+48-(32)-2371818$ (S.B.)
}

Citation: Chrobok, L.; Bajkacz, S.; Klich, J.D.; Lewandowski, M.H. LC-MS/MS Analysis Elucidates a Daily Rhythm in Orexin A Concentration in the Rat Vitreous Body. Molecules 2021, 26, 5036. https://doi.org/10.3390/ molecules26165036

Academic Editor: James Barker

Received: 7 July 2021

Accepted: 16 August 2021

Published: 19 August 2021

Publisher's Note: MDPI stays neutral with regard to jurisdictional claims in published maps and institutional affiliations.

Copyright: (c) 2021 by the authors. Licensee MDPI, Basel, Switzerland. This article is an open access article distributed under the terms and conditions of the Creative Commons Attribution (CC BY) license (https:// creativecommons.org/licenses/by/ $4.0 /)$.

\begin{abstract}
Orexins are two neuropeptides synthesised mainly in the brain lateral hypothalamic area. The orexinergic system provides arousal-dependent cues for a plethora of brain centres, playing a vital role in feeding behaviour, regulation of the sleep-wake cycle and circadian rhythms. Recently, orexins were found to be produced in the retina of an eye; however, their content in the vitreous body and possible daily pattern of expression have not yet been explored. In this manuscript, we describe the development and validation of a liquid chromatography with tandem mass spectrometry (LCMS/MS) method designed for quantitative bioanalysis of orexin in the rat vitreous body. Orexin was extracted from vitreous body samples with a water:acetonitrile:formic acid (80:20:0.1; $v / v / v)$ mixture followed by vortexing and centrifuging. Separation was performed on a reverse-phase HPLC column under gradient conditions. Orexin was analysed via multiple-reaction monitoring (MRM) in the positive electrospray mode. The total analysis time for each sample was less than $5.0 \mathrm{~min}$. Once the method was fully optimised, it was then validated, following the 2018 FDA guidance on bioanalytical method validations. The calibration curves for orexin $(1-500 \mathrm{ng} / \mathrm{mL})$ were constructed using a linear regression with a $1 / \mathrm{x}^{2}$ weighting. The lower limit of quantitation for orexin was $1.0 \mathrm{pg} / \mathrm{mL}$ for the vitreous body. Intra-day and inter-day estimates of accuracy and precision were within $10 \%$ of their nominal values, indicating that the method is reliable for quantitation of orexin in the rat vitreous body. From the physiological perspective, our results are the first to show daily rhythm of orexin synthesis by the retina with possible implications on the circadian regulation of vision.
\end{abstract}

Keywords: orexin; vitreous body; LC-MS/MS; circadian clock

\section{Introduction}

The rotation of Earth around its axis within a period of $24 \mathrm{~h}$ evokes pronounced cyclic environmental alterations of day and night. Living organisms had to adapt to these rhythmic changes, and the ability to anticipate them created a huge evolutionary advantage. Thus, nearly all life on Earth possesses intrinsic timekeeping mechanisms called circadian clocks. In mammals, the master clock is localised in the hypothalamic brain structure-the suprachiasmatic nuclei (SCN) [1,2]. However, accumulating evidence shows the existence of local clocks in other brain areas and peripheral tissues, such as the liver, kidney, lungs [3-6], or the retina of an eye [7-9]. Due to a modern 24/7 lifestyle, shift work or during the course of jet lag, body clocks desynchronise, leading to severe civilisational health problems, including obesity, cardiovascular diseases and some types of cancer [10]. Thus, understanding circadian rhythmicity requires further thorough exploration, including the involvement of new analytical tools for chronobiological investigation. 
Orexins are two neuropeptides (orexin A and B, OXA and OXB, respectively) synthesised predominately in the lateral hypothalamic area as a product of proteolysis of their common precursor-prepro-orexin [11-13]. The molecular masses of OXA (33 amino acids) and OXB (28 amino acids) are 3562 and $2937 \mathrm{Da}$, respectively. In contrast to OXB, OXA contains four cysteine residues that form two intrachain disulphide bonds. In the brain, the orexinergic system has been hypothesised to act as the hands of the master clock, providing timed arousal-dependent cues for the plethora of neuronal centres. Due to its reciprocal connections with the $\mathrm{SCN}$, the neuronal activity of orexinergic neurons in rodents follows the light-dark cycle, with higher activity seen during the behaviourally active night $[14,15]$. It has been additionally shown that orexins are produced and act locally in the retina of rodents and humans [16-20]. A recent report demonstrated retinal orexins to control the pupillary light reflex [21]. However, the temporal coordination of orexin release in the retina on the daily timescale remained elusive.

To verify the above hypotheses, it is necessary to develop an analytical method for the determination of orexin in the vitreous body, a gel-like fluid filling the vitreous chamber of the eyeball ranging from the lens to the retina. Due to the close proximity to retinal cells, the vitreous body not only provides a clear pathway for light to reach the photoreceptors but also creates a reservoir for many substances synthesised by the retina [22,23]. Only three analytical methods for the determination of orexin have been published to date. Two of these methods for quantification of OXA in the cerebrospinal fluid are based on liquid chromatography with tandem mass spectrometry (LC-MS/MS) analysis and standard radioimmunoassay $[24,25]$. In the third paper, the authors focus on research on reducing adsorption to improve recovery and in vivo detection of OXA and OXB by microdialysis with LC-MS [26]. These methods were applied only for determination of orexin in the cerebrospinal fluid, and no simultaneous analysis of the vitreous body has been described hitherto.

Therefore, the aim of the study was to detect and quantify OXA content in the rat vitreous body with the use of LC-MS/MS analytical methods. Here, we show that OXA may be reliably detected from vitreous body samples, which proves their local expression in the retina. Additionally, our study provides compelling evidence for daily fluctuations in the OXA content in the eye, which opens new pathways for further physiological and pharmacological studies.

\section{Materials and Methods}

\subsection{Chemicals and Reagents}

Orexin A (OXA) (purity 97\%) was purchased from Bachem (Bubendorf, Switzerland). Acetonitrile and methanol (HPLC-MS grade), water and formic acid (HPLC grade) were purchased from Merck (Darmstadt, Germany). The stock solution of OXA was prepared in methanol at a $1.0 \mathrm{mg} / \mathrm{mL}$ concentration. Stock solutions were further diluted with methanol to obtain OXA working solutions at several concentration levels.

Calibration standards (CS) and quality control (QC) samples in the rat vitreous body were prepared daily by diluting the corresponding working solutions with an OXA-free (blank) rat vitreous body. Before preparing the CS and QC samples, the rat vitreous body applied as a blank sample was examined to ensure that it was free of OXA. The CS and QC samples were prepared on each analysis day with the procedures described below. The concentrations of the CS were 1, 10, 20, 50, 100, 200, 350 and $500 \mathrm{pg} / \mathrm{mL}$ of OXA in the rat vitreous body. The QC samples in the rat vitreous body were prepared at three concentrations: lower limit of quantitation (LLOQ): $1 \mathrm{pg} / \mathrm{mL}$; low concentration quality control (LQC): $10 \mathrm{pg} / \mathrm{mL}$; middle concentration quality control (MQC): $100 \mathrm{pg} / \mathrm{mL}$; and high concentration quality control (HQC): $500 \mathrm{pg} / \mathrm{mL}$. All stock, standard working and QC working solutions were stored at $4{ }^{\circ} \mathrm{C}$ and were brought to room temperature before use. Quantitative analysis of the OXA was performed for the rat vitreous body samples; therefore, the validation of the method was also performed for this matrix (CS and QC samples prepared in the rat vitreous body). 


\subsection{Ethical Approval}

Animals were maintained and used according to Polish regulations and the European Communities Council Directive (86/609/EEC). All procedures were approved by the Local Ethics Committee in Krakow and all efforts were made to minimise the number of animals used and their suffering.

\subsection{Animals}

This study was performed on 20 adult (8-10 week old) Sprague-Dawley rats of both sexes. Rats were bred and housed in the Animal Facility at the Institute of Zoology and Biomedical Research, Jagiellonian University in Krakow, three to six per cage at $23 \pm 2{ }^{\circ} \mathrm{C}$ and $67 \pm 3 \%$ relative humidity. Animals were provided with ad libitum access to food and water and kept under standard 12:12 h light-dark (LD) cycle. All procedures in darkness were performed under dim red light, not visible to rodents.

\subsection{Tissue Collection}

Rats were deeply anaesthetised with isoflurane inhalation ( $2 \mathrm{~mL}$ per chamber) and intracardially injected with an overdose of sodium pentobarbital $(100 \mathrm{mg} / \mathrm{kg}$ body weight; Biowet, Pulawy, Poland). Following this, the eyes were enucleated, rinsed in distilled water and quickly dried with a disposable tissue paper. Then, the eyes were hemisected with a fresh surgical blade, and the vitreous body $(20 \mu \mathrm{L})$ was collected in a fresh test-tube. Samples from both eyes were pooled together, flash frozen over dry ice and kept at $-80^{\circ} \mathrm{C}$.

Culls were performed at four daily time points ( $n=5$ rats per group), at Zeitgeber time (ZT) 1, 7, 13 and 19, where ZT0 means the time of lights-on and ZT12 means lights-off. Procedures at ZT1 and ZT7 were performed in ambient light ( 300 lux), whereas those at ZT13 and ZT19 were carried out under dim red light.

\subsection{Sample Preparation}

A $10 \mu \mathrm{L}$ sample aliquot (rat vitreous body) was placed in a $1.5 \mathrm{~mL}$ microcentrifuge tube. The sample was extracted with $90 \mu \mathrm{L}$ of mixture water:acetonitrile:formic acid (80:20:0.1; $v / v / v$ ) by vortex mixing for $10 \mathrm{~min}$. The supernatant was collected after centrifugation at $15,000 \times g$ for $10 \mathrm{~min}$. Ten microlitres of the supernatant was injected into the LC-MS/MS system for analysis.

\subsection{LC-MS/MS Analysis}

LC-MS/MS analysis was performed on a Dionex UHPLC system (Dionex Corporation, Sunnyvale, CA, USA) coupled to a QTRAP 4000 triple quadrupole linear ion trap mass spectrometer equipped with an electrospray ionisation (ESI) interface (Applied Biosystems/MDS SCIEX, Foster City, CA, USA). The LC system includes an autosampler, a binary pump, a compartment with a thermostable column area and a variable wavelength detector. The data were acquired using Analyst software, Version 1.4.

OXA analysis was carried out on a Kinetex C18 analytical column $(75 \times 2.1 \mathrm{~mm}$; $2.6 \mu \mathrm{m}$ ) with column temperature set at $30^{\circ} \mathrm{C}$. The mobile phase consisted of $0.1 \%$ formic acid in water (mobile phase A) and acetonitrile (mobile phase B). Sample separation was conducted under gradient conditions with a flow rate of $0.8 \mathrm{~mL} / \mathrm{min}$. The initial elution composition was $90 \%(v / v)$ mobile phase A, followed by a linear gradient up to $65 \%(v / v)$ mobile phase A at $3 \mathrm{~min}$ and returning back to initial composition at $3.1 \mathrm{~min}$. The column equilibration time was $1.9 \mathrm{~min}$, leading to a total run time of $5 \mathrm{~min}$. The injection volume was $5 \mu \mathrm{L}$. The autosampler's temperature was set at $10{ }^{\circ} \mathrm{C}$.

To select the MS/MS parameters, a standard solution of OXA at concentration $500 \mathrm{ng} / \mathrm{mL}$ was infused into the mass spectrometer using a Harvard syringe pump at a flow rate of $10 \mu \mathrm{L} / \mathrm{min}$. Finally, multiple reaction monitoring (MRM) mode was applied for the detection of OXA by monitoring the transitions: MRM1 (quantifier) $m / z 713.3 \rightarrow 858.6$ and MRM2 (qualifier) $m / z 713.3 \rightarrow 854.1$ with a dwell time of $250 \mathrm{~ms}$ for each transition. The collision energy was optimised to $22 \mathrm{~V}$ and $25 \mathrm{~V}$ for MRM1 and MRM2, respectively. Other compound- 
dependent parameters (declustering potential (DP), collision energy (CE), entrance potential (EP) and collision cell exit potential (CXP)) were as follows: $100 \mathrm{~V} ; 10 \mathrm{~V} ; 7 \mathrm{~V}$. The IonSpray voltage was set at $5500 \mathrm{~V}$ in the positive ionisation mode. The source temperature was $500{ }^{\circ} \mathrm{C}$. Nitrogen (nebulising gas) pressure, turbo spray gas pressure, curtain gas and collision gas pressure were set at 50 psi, 40 psi, 20 psi and 5 psi, respectively.

\subsection{Method Validation}

Selectivity: Selectivity assessment was performed in chromatograms of blank rat vitreous body samples compared to those with an OXA spike to explore the potential interference with the analyte.

Linearity and LLOQ: The linearity test was conducted by injecting eight calibration standard solutions of concentrations ranging from 1 to $500 \mathrm{pg} / \mathrm{mL}$ of OXA in the rat vitreous body and fitted to $y=b x+c$ by a weighting factor of $1 / x^{2}$. The lower limit of quantification (LLOQ) was defined as the lowest calibration curve concentration, at which the deviation of accuracy (RE) was within $\pm 20 \%$ and precision (RSD) $<20 \%$. The signal-to-noise ratio $(\mathrm{S} / \mathrm{N})$ was more than 10.

Precision and accuracy: Precision and accuracy were calculated as relative standard deviation (\%RSD) and relative error (\%RE) from the theoretical/nominal values, respectively. Three replicates of each QC sample, namely, LLOQ $=1 \mathrm{pg} / \mathrm{mL}, \mathrm{LQC}=10 \mathrm{pg} / \mathrm{mL}$, $\mathrm{MQC}=100 \mathrm{pg} / \mathrm{mL}$ and $\mathrm{HQC}=400 \mathrm{pg} / \mathrm{mL}$, were analysed on the same day (inter-day) and on different days (intra-day) with the calibration curve to determine the precision and accuracy of the method. The deviation in the precision (\%RSD) and accuracy (\%RE) values was limited to $\leq 15 \%$ for the QC samples except for LLOQ samples, where it was limited to $\pm 20 \%$ ( $\%$ RSD and $\%$ RE).

Matrix effect and recovery: To evaluate the matrix effect (ME), blank matrices were extracted and spiked with the QC samples (post-spike sample). The ME was calculated by comparing the peak area of OXA in post-spike samples with that in external (unextracted) samples at the same concentration. The extraction recovery (ER) of OXA from the rat vitreous body was evaluated by comparing the mean area of the extracted QCs with that of samples at the same concentration obtained by spiking the standard solution with the extracted blank matrix samples.

Stability: The stability of the analytes at different storage conditions was also assessed at four concentration levels (LLOQ, LQC, MQC and HQC). Short-term and long-term stabilities were evaluated for spiked rat vitreous body samples kept in the autosampler for up to $24 \mathrm{~h}\left(10^{\circ} \mathrm{C}\right)$, at $-80{ }^{\circ} \mathrm{C}$ for one week and at $-20^{\circ} \mathrm{C}$ for one month, respectively. Freeze-thaw stability was also assessed in three freeze-thaw cycles. Spiked QC samples were kept frozen at $-80{ }^{\circ} \mathrm{C}$ overnight and thawed at room temperature the following day. The third day QCs were thawed, prepared and analysed, as described previously. All analysed samples were compared to freshly prepared matched QC samples.

Carry-over effect: The carry-over effect was evaluated by injecting blank rat vitreous body samples and methanol, respectively, after the analysis of samples spiked at $1000 \mathrm{pg} / \mathrm{mL}$ OXA.

Dilution integrity: Dilution integrity was demonstrated using samples spiked at a concentration of $1000 \mathrm{pg} / \mathrm{mL}$, which were diluted to $1 / 10$ of their concentration with a blank rat vitreous body. Dilution integrity was determined with six replicates.

\subsection{Statistics}

First, data triplicates were averaged for each animal $(n=$ an average of three analyses from one vitreous body sample). SD was measured for these animal-averaged values, thus demonstrating inter-subject differences. Statistical testing based on $\mathrm{nM}$ concentration values was performed in Prism 7 (GraphPad Software, San Diego, CA, USA) with ordinary one-way ANOVA. Normality of the distribution was assessed with the KolmogorovSmirnov test with Dallal-Wilkinson-Lilliefor p-values. Data were then transformed to relative orexin content ( $\% \mathrm{ZT} 1)$ by calculating the $\%$ value for each data point in relation to 
ZT1 average (set at 100\%). Next, relative values were tested for rhythmicity in CircWave v1.4 software (developed by Dr Roelof Hut, University of Groningen, The Netherlands, http: / / www.euclock.org/results /item/circ-wave.html (accessed on 23 June 2021)). All data are presented as mean \pm standard deviation (SD), and $p<0.05$ was deemed significant.

\section{Results and Discussion}

\subsection{LC-MS/MS Method Development}

The MS/MS parameters were optimised to produce the maximum response for the orexin standard. The MS signal was optimised in full scan mode in the $m / z$ range of 100-900 under positive ionisation. The protonated molecular ion $[\mathrm{M}+5 \mathrm{H}]^{5+}$ with $\mathrm{m} / z$ 713 was identified as the major peak for the selected peptide. The two most intense fragment ions observed were the ions with $m / z 858.6$ and $m / z$ 854.1. The ion with $m / z$ 858.6 was selected since it was produced in relatively greater abundance, which was also reported in previous studies [25]. The analyte-dependent parameters, especially collision energy, were optimised for each transition in order to obtain the most sensitive method (Section 2.6).

The HPLC conditions were optimised to eliminate interferences from the matrix and to elute the analyte within a short run time $(5.0 \mathrm{~min})$. To obtain the best chromatographic conditions, the mobile phase composition, type of column, column temperature and flow rate were judiciously selected. The Kinetex C18 column was found to be optimal compared with the tested columns, including Chromolith ${ }^{\circledR}$ Fast Gradient Monolithic C18e, Hypersil Gold and Kinetex F5 due to the unique, superficially porous particles and $2.6 \mu \mathrm{m}$ particle size providing high resolution of the tested analyte from matrix components. In addition, this column enabled a robust symmetrical peak to be obtained and a more sensitive method to be used compared to other columns.

The feasibility of using various mixtures of solvents, such as acetonitrile and methanol, and different additives, such as ammonium acetate, ammonium formate, acetic acid and formic acid, with a variable $\mathrm{pH}$ range of 2.5-5.5, along with altered flow rates (in the range of $0.3-1.0 \mathrm{~mL} / \mathrm{min}$ ), was tested for the complete chromatographic resolution of OXA and matrix components. The optimal mobile phase was found to consist of acetonitrile and water containing $0.1 \%(v / v)$ formic acid $(\mathrm{pH}=3.0)$. A gradient elution was applied to improve the separation of OXA from the eluting interferences and to facilitate efficient ionisation of the analyte under electrospray conditions. Acetonitrile rather than methanol was chosen as the organic modifier because acetonitrile led to lower background noise and resulted in the best resolution. During the early stages of method development, a small amount of acidic modifier $(0.1 \%(v / v)$ formic acid) was added to the mobile phase to improve the MS/MS response of the analyte of interest. Moreover, the effects of column temperature were investigated in the range of $20-45^{\circ} \mathrm{C}$. The retention time decreased slightly, and no change in peak shape was observed with increasing temperature. Therefore, the temperature was maintained at $30^{\circ} \mathrm{C}$. The developed LC conditions employed in the present method resulted in a retention time of $3 \mathrm{~min}$ for OXA (Figure 1). Additionally, representative chromatograms obtained in MRM acquisition mode for OXA at three concentration levels are shown in Figure S1 (Supplementary Material).

Mixtures of methanol, acetonitrile, water, formic acid and acetic acid were used for the extraction under acidic to neutral $\mathrm{pH}$ conditions. The lowest recovery of OXA, 10\%-30\%, was obtained for pure water. Adding acetonitrile to samples increased the signal for the studied peptide. We investigated the effect of acetonitrile added at different concentrations $(5 \%-100 \%)$. Optimal acetonitrile concentrations were from $15 \%$ to $25 \%$. Increasing the amount of organic phase in the extraction mixture was found to significantly lower its extraction efficiency. Extraction with pure acetonitrile gave recoveries ranging from 50\% to $60 \%$. The addition of acetonitrile to water in a 1:1 volume ratio increased the recovery of OXA by $20 \%$ compared to pure acetonitrile. The highest recovery was obtained for the mixture of water:acetonitrile:formic acid $(80: 20: 0.1 ; v / v / v)$. The conversion of acetonitrile to methanol caused the extraction yield to drop by $30 \%$ due to the higher polarity of the 
mixture. The use of the acetic acid instead of formic acid resulted in an increase in OXA recovery (70\%). Summarising, a solvent mixture of water:acetonitrile:formic acid (80:20:0.1; $v / v / v$ ) was identified to affect maximal extraction of orexin from vitreous body samples.

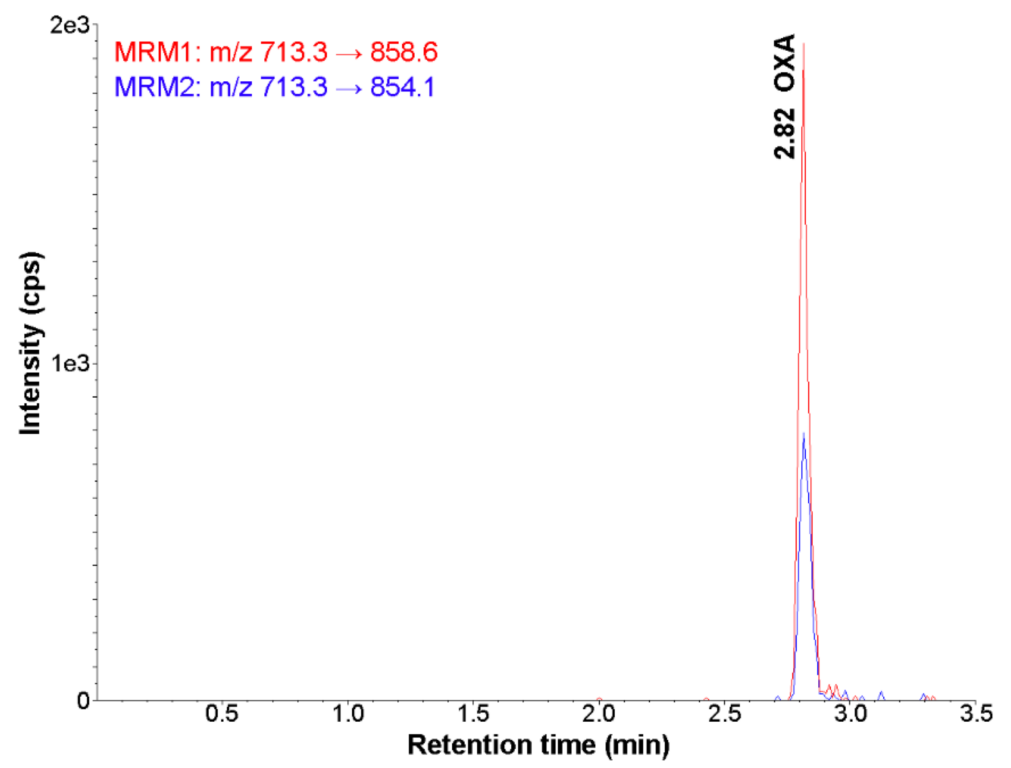

Figure 1. Representative MRM chromatogram of orexin (standard solution at concentration $100 \mathrm{pg} / \mathrm{mL}$ ) (UHPLC-MS/MS method).

\subsection{Method Validation}

This method was validated and included all the performance criteria needed, such as selectivity, linearity, sensitivity, accuracy, precision, recovery, matrix effect, carry-over effect and dilution integrity.

Selectivity: For the blank vitreous body, there was a minor peak observed with a peak area of less than $20 \%$ of the LLOQ area. Based on the consistently low intensity of this signal, it was determined that the endogenous peak would not substantially affect the accurate quantitation of OXA. Exemplarily, MRM chromatograms of the extract of the vitreous body spiked with $1 \mathrm{pg} / \mathrm{mL}$ OXA and in the extract of the blank vitreous body are presented in Figure 2.
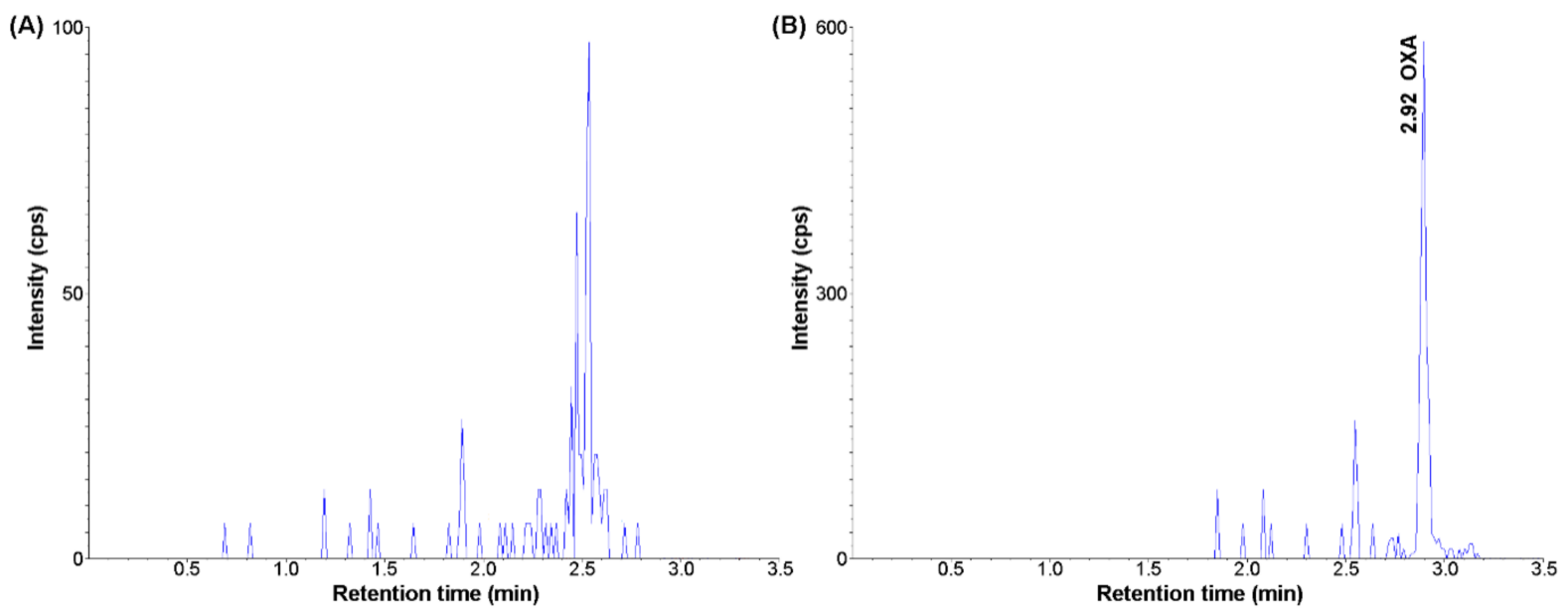

Figure 2. Representative MRM chromatograms of orexin A: (A) blank vitreous body; (B) blank vitreous body spiked with orexin A (1 pg/mL) (UHPLC-MS/MS method). 
Linearity and LLOQ: The calibration curves showed good linearity $(r>0.9921)$ over the tested concentration ranges of OXA in the rat vitreous body samples. The method showed good sensitivity at the level of $1 \mathrm{pg} / \mathrm{mL}(\mathrm{S} / \mathrm{N}>10)$ for OXA in the rat vitreous body samples, which were sufficient to study the orexin content in the eye.

Precision and accuracy: The calculated results of intra-day and inter-day precision as well as accuracy data for OXA (at four concentration levels of QC samples) in the rat vitreous body are represented in Table 1 . The intra-day precision (\%RSD) was found in the range of $2.8 \%$ to $5.2 \%$, and accuracy (\%RE) was in the range of $-6.8 \%$ to $-2.0 \%$. Similarly, the inter-day precision was found in the range of $4.4 \%$ to $6.5 \%$, and accuracy was in the range of $-9.1 \%$ to $-5.7 \%$, which indicates that the developed LC-MS/MS method is suitable for the analysis of OXA in rat vitreous body samples.

Table 1. Intra-day and inter-day precision and accuracy, extraction recovery and matrix effect for OXA-spiked rat vitreous body $(n=6)$.

\begin{tabular}{ccccccc}
\hline \multirow{2}{*}{$\begin{array}{c}\text { Concentration Added } \\
(\mathbf{p g} / \mathbf{m L})\end{array}$} & \multicolumn{2}{c}{$\begin{array}{c}\text { Precision } \\
\text { RSD (\%) }\end{array}$} & \multicolumn{2}{c}{$\begin{array}{c}\text { Accuracy } \\
\text { RE (\%) }\end{array}$} & $\begin{array}{c}\text { Matrix Effect } \\
\text { ME (\%) }\end{array}$ & $\begin{array}{c}\text { Extraction Recovery } \\
\text { ER (\%) }\end{array}$ \\
\hline 1 & 5.2 & 6.5 & -6.8 & -9.1 & 110.2 & 95.4 \\
Intra-Day & Inter-Day & Intra-Day & Inter-Day & -107.5 & 97.1 \\
10 & 3.7 & 5.2 & -3.6 & -8.3 & 107.2 & 97.8 \\
40 & 3.1 & 5.0 & -2.1 & -7.3 & 102.1 & 98.3 \\
\hline
\end{tabular}

Matrix effect and recovery: Reasonable values of ME ranging from $102.1 \%$ to $110.2 \%$ were obtained for quality control samples that were spiked at four concentrations: LLOQ, LQC, MQC and HQC. Adequate ER values ranging from $95.4 \%$ to $98.3 \%$ were obtained for OXA at four concentration levels (Table 1).

Stability: The stability data are presented in Table 2. In all the stability tests, the analytes showed good stability with RE values ranging from $-9.8 \%$ to $-2.9 \%$, and $C V$ values were $<8.3 \%$ at different storage conditions (autosampler, short-term and long-term stability, freeze-thaw stability) suggesting no significant degradation.

Table 2. Stability results of OXA in rat vitreous body in different conditions $(n=6)$.

\begin{tabular}{|c|c|c|c|c|c|c|c|c|}
\hline \multirow{2}{*}{$\begin{array}{c}\text { Concentration } \\
\text { Added } \\
(\mathrm{pg} / \mathrm{mL})\end{array}$} & \multicolumn{2}{|c|}{ Autosampler Stability } & \multicolumn{2}{|c|}{ Short-Term Stability } & \multicolumn{2}{|c|}{ Long-Term Stability } & \multicolumn{2}{|c|}{ Freeze-Thaw Stability } \\
\hline & $\begin{array}{c}\text { RSD } \\
(\%)\end{array}$ & $\begin{array}{l}\text { RE } \\
(\%)\end{array}$ & $\begin{array}{c}\text { RSD } \\
(\%)\end{array}$ & $\begin{array}{l}\text { RE } \\
(\%)\end{array}$ & $\begin{array}{c}\text { RSD } \\
(\%)\end{array}$ & $\begin{array}{l}\text { RE } \\
(\%)\end{array}$ & $\begin{array}{c}\text { RSD } \\
(\%)\end{array}$ & $\begin{array}{l}\text { RE } \\
(\%)\end{array}$ \\
\hline 1 & 4.8 & -6.4 & 5.8 & -6.9 & 5.2 & -9.8 & 8.3 & -9.1 \\
\hline 10 & 4.4 & -5.6 & 4.8 & -6.3 & 4.4 & -8.4 & 3.2 & -8.9 \\
\hline 100 & 2.8 & -5.5 & 4.0 & -4.5 & 3.1 & -5.7 & 2.5 & -5.7 \\
\hline 400 & 1.6 & -3.2 & 2.3 & -3.9 & 2.1 & -2.9 & 1.9 & -4.5 \\
\hline
\end{tabular}

Carry-over effect: Carry-over in the first double blank after ULOQ determined in three runs for OXA was $1.3 \%$ to $6.6 \%$ of the LLOQ mean peak area. The acceptance criteria were met. Carry-over is expected not to have any impact on study sample results. Nevertheless, carry-over was monitored and evaluated during study sample analysis in each batch by injecting double blanks/blanks after ULOQ.

Dilution integrity: One tenfold dilution of a $1000 \mathrm{ng} / \mathrm{mL}$ dilution $\mathrm{QC}$ sample $(\mathrm{n}=6)$ resulted in accuracy and precision of $1.6 \%$ and $2.5 \%$, respectively, for the rat vitreous body samples, thus meeting the acceptance criteria.

All results obtained during method validation met the acceptance criteria of the US-FDA Guideline for Bioanalytical Validation (2018) [27].

\subsection{Method Applicability}

The method described above was used to quantify OXA concentrations in the biological samples across the daily cycle. Twenty samples of the vitreous body were collected 
at four time points across $24 \mathrm{~h}(\mathrm{ZT} 1,7,13,19$, where ZT0 means the time of lights-on and ZT12means lights off). Each sample was analysed in triplicate, and the obtained results were averaged for each animal. Figure 3 shows the MRM chromatogram of the extract of the rat vitreous body after LC-MS/MS analysis. The average OXA concentration in the vitreous body for all samples amounted to $13.09 \pm 3.5 \mathrm{nM}(\mathrm{n}=20)$, which stands in the physiological range of OXA action upon orexin receptors $\left(\mathrm{IC}_{50}=20\right.$ and $38 \mathrm{nM}$ for orexin receptors 1 and 2, respectively [11]).

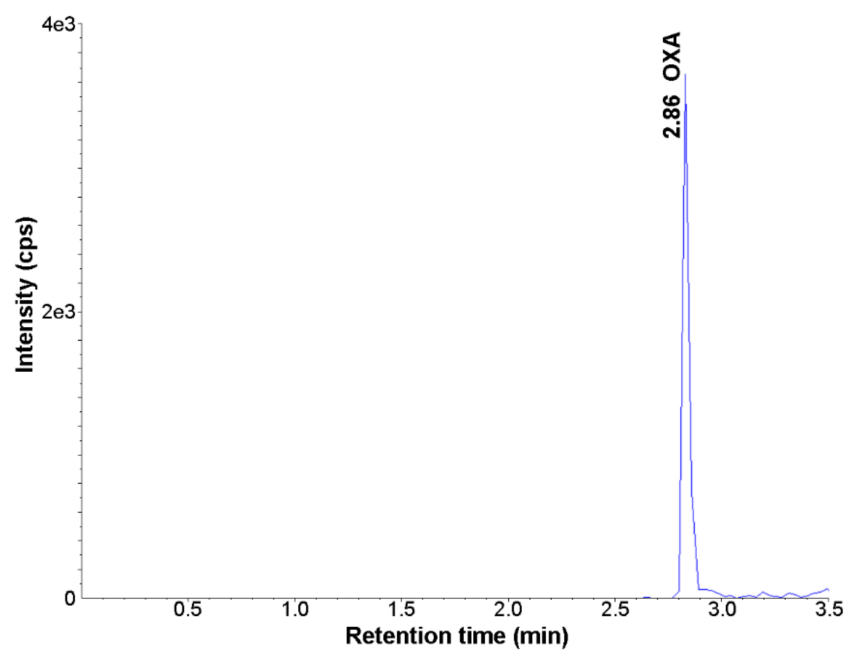

Figure 3. Representative MRM chromatogram of orexin A in the rat vitreous body sample (MRM1; $m / z 713.3 \rightarrow 858.6$ ) (UHPLC-MS/MS method).

However, further analysis proved that OXA concentration is subject to daily variation $(p=0.0002, \mathrm{n}=20$, one-way ANOVA; Figure 4A). It stayed in relatively high values during the night to the beginning of the light phase, with a notable drop in the middle of the day (reaching $53.02 \pm 9.1 \%$ of ZT1 mean). Next, the rhythmicity of this daily pattern was assessed by sine curve fitting. Indeed, the analysis proved the distribution to be rhythmic $(p=0.049, \mathrm{n}=20$, CircWave; Figure 4B), with an acrophase of the rhythm at ZT19.9. This dataset shows that OXA may be reliably quantified in the rat vitreous body samples, showing physiologically relevant concentrations. Additionally, it shows a significant daily rhythmicity of OXA content peaking in the middle of the night and exhibiting a notable drop during the behaviourally quiescent day. This daily pattern resembles the brain orexin rhythm in the lateral hypothalamus and subcortical visual structures $[15,28,29]$.
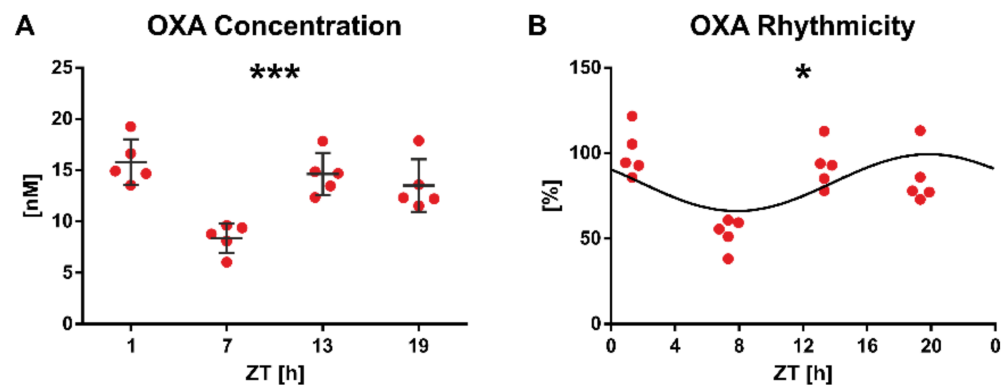

Figure 4. Orexin A (OXA) exhibits daily rhythmicity in the rat vitreous body samples. (A) Daily variation in concentration assessed by one-way ANOVA (*** $p=0.0002, \mathrm{n}=20$ ). (B) Daily rhythmicity studied with sine wave fitting ( ${ }^{*} p=0.049, \mathrm{n}=20$; CircWave). Each data point demonstrates an average of three measurements obtained from one vitreous body collected from one animal. \%—relative OXA concentration to ZT1 average. ZT-Zeitgeber time. Data are presented as a mean $\pm \mathrm{SD}$. 


\section{Conclusions}

The present study reports the development and validation of a sensitive and specific LC-MS/MS method for the quantification of OXA in the rat vitreous body. Advantages include a chromatography run time of $5 \mathrm{~min}$ and the use of a small volume of sample $(10 \mu \mathrm{L})$ with improved analytical sensitivity $(1 \mathrm{pg} / \mathrm{mL})$. The method was designed according to the current FDA bioanalytical guidance requirements as fit for purpose. Moreover, this report is the first to provide a quantitative description of OXA presence in the eye vitreous body in concentrations sufficient to functionally activate orexin receptors. Importantly, retinal-derived orexin content followed a daily pattern, with a peak in the middle of the behaviourally active night. This suggests either the retinal clock-dependent expression of the prepro-orexin gene or regulation of expression/secretion via the light-dark cycle. These findings, possible due to the utilisation of our LC-MS/MS method, provide valuable physiological background to the orexinergic modulation of early visual processing.

Supplementary Materials: The following are available online, Figure S1: Representative MRM chromatograms of orexin A at different concentrations ((A) 10 pg/ml; (B) 100 pg/ml; (C) 400 pg/ml) (UHPLC-MS/MS).

Author Contributions: L.C. conceived the study and provided financial support. L.C. and M.H.L. supervised the study. S.B. and L.C. designed the protocols and interpreted the results of the study. J.D.K. and L.C. harvested biological samples. S.B. performed measurements and analysed the data. S.B. and L.C. wrote the paper, and all authors agreed to the final version. All authors have read and agreed to the published version of the manuscript.

Funding: This work was financially supported by the project 'Sonatina 2' 2018/28/C/NZ4/00099 given to L.C.

Institutional Review Board Statement: This study was performed according to Polish regulations and the European Communities Council Directive of 24 November 1986 (86/609/EEC). All procedures performed on rats were approved by the Local Ethics Committee in Krakow (253/2018 approval date: 13.09.2018).

Informed Consent Statement: Not applicable.

Data Availability Statement: The data that support the findings of this study are available from the corresponding authors upon reasonable request.

Conflicts of Interest: The authors declare no competing financial interest.

Sample Availability: Samples of the compounds are not available from the authors.

\section{References}

1. Hastings, M.H.; Maywood, E.S.; Brancaccio, M. The mammalian circadian timing system and the suprachiasmatic nucleus as its pacemaker. Biology 2019, 8, 13. [CrossRef]

2. Hastings, M.H.; Maywood, E.S.; Brancaccio, M. Generation of circadian rhythms in the suprachiasmatic nucleus. Nat. Rev. Neurosci. 2018, 19, 453-469. [CrossRef]

3. Fukuhara, C. Peripheral circadian oscillators and their rhythmic regulation. Front. Biosci. 2003, 8, d642. [CrossRef] [PubMed]

4. Brown, A.J.; Pendergast, J.S.; Yamazaki, S. Peripheral circadian oscillators. Yale J. Biol. Med. 2019, 92, 327-335. Available online: http:/ / www.ncbi.nlm.nih.gov/pubmed/31249493 (accessed on 23 June 2021). [PubMed]

5. Begemann, K.; Neumann, A.; Oster, H. Regulation and function of extra-SCN circadian oscillators in the brain. Acta Physiol. 2020, 229, e13446. [CrossRef]

6. Guilding, C.; Piggins, H.D. Challenging the omnipotence of the suprachiasmatic timekeeper: Are circadian oscillators present throughout the mammalian brain? Eur. J. Neurosci. 2007, 25, 3195-3216. [CrossRef] [PubMed]

7. Ruan, G.-X.; Zhang, D.-Q.; Zhou, T.; Yamazaki, S.; McMahon, D.G. Circadian organization of the mammalian retina. Proc. Natl. Acad. Sci. USA 2006, 103, 9703-9708. [CrossRef]

8. Guido, M.E.; Garbarino-Pico, E.; Contin, M.A.; Valdez, D.J.; Nieto, P.S.; Verra, D.M.; Acosta-Rodriguez, V.; de Zavalía, N.; Rosenstein, R.E. Inner retinal circadian clocks and non-visual photoreceptors: Novel players in the circadian system. Prog. Neurobiol. 2010, 92, 484-504. [CrossRef] [PubMed]

9. McMahon, D.G.; Iuvone, P.M.; Tosini, G. Circadian organization of the mammalian retina: From gene regulation to physiology and diseases. Prog. Retin. Eye Res. 2014, 39, 58-76. [CrossRef]

10. Baron, K.G.; Reid, K.J. Circadian misalignment and health. Int. Rev. Psychiatry 2014, 26, 139-154. [CrossRef] 
11. Sakurai, T.; Amemiya, A.; Ishii, M.; Matsuzaki, I.; Chemelli, R.M.; Tanaka, H.; Williams, S.C.; Richardson, J.A.; Kozlowski, G.P.; Wilson, S.; et al. Orexins and orexin receptors: A family of hypothalamic neuropeptides and G protein-coupled receptors that regulate feeding behavior. Cell 1998, 199892, 573-585. Available online: http:/ / www.ncbi.nlm.nih.gov/pubmed/9491897 (accessed on 23 June 2021). [CrossRef]

12. de Lecea, L.; Kilduff, T.S.; Peyron, C.; Gao, X.-B.; Foye, P.E.; Danielson, P.E.; Fukuhara, C.; Battenberg, E.L.F.; Gautvik, V.T.; Bartlett, F.S.; et al. The hypocretins: Hypothalamus-specific peptides with neuroexcitatory activity. Proc. Natl. Acad. Sci. USA 1998, 95, 322-327. [CrossRef] [PubMed]

13. Li, S.-B.; de Lecea, L. The hypocretin (orexin) system: From a neural circuitry perspective. Neuropharmacology 2020, 167, 107993. [CrossRef]

14. Azeez, I.A.; del Gallo, F.; Cristino, L.; Bentivoglio, M. Daily fluctuation of orexin neuron activity and wiring: The challenge of "chronoconnectivity". Front. Pharmacol. 2018, 9, 1061. [CrossRef]

15. Marston, O.J.; Williams, R.H.; Canal, M.M.; Samuels, R.E.; Upton, N.; Piggins, H.D. Circadian and dark-pulse activation of orexin/hypocretin neurons. Mol. Brain 2008, 1, 19. [CrossRef] [PubMed]

16. Savaskan, E.; Müller-Spahn, F.; Meier, F.; Wirz-Justice, A.; Meyer, P. Orexins and their receptors in the human retina. Pathobiology 2004, 71, 211-216. [CrossRef] [PubMed]

17. Qiao, S.; Zhou, W.; Liu, L.; Zhang, D.; Zhong, Y. Orexin-A suppresses signal transmission to dopaminergic amacrine cells from outer and inner retinal photoreceptors. Investig. Ophthalmol. Vis. Sci. 2017, 58, 4712. [CrossRef]

18. Zhang, G.; Wu, X.-H.; Xu, G.-Z.; Weng, S.-J.; Yang, X.-L.; Zhong, Y.-M. Orexin-B modulates synaptic transmission of rod bipolar cells in rat retina. Neuropharmacology 2018, 133, 38-50. [CrossRef]

19. Ruan, H.-Z.; Wang, L.-Q.; Yuan, F.; Weng, S.-J.; Zhong, Y.-M. Orexin-A differentially modulates inhibitory and excitatory synaptic transmission in rat inner retina. Neuropharmacology 2021, 187, 108492. [CrossRef]

20. Liu, F.; Xu, G.Z.; Wang, L.; Jiang, S.X.; Yang, X.L.; Zhong, Y.M. Gene expression and protein distribution of orexins and orexin receptors in rat retina. Neuroscience 2011, 189, 146-155. [CrossRef] [PubMed]

21. Zhou, W.; Wang, L.-Q.; Shao, Y.-Q.; Han, X.; Yu, C.-X.; Yuan, F.; Wang, X.; Weng, S.-J.; Zhong, Y.-M.; Yang, X.-L. Orexin-A intensifies mouse pupillary light response by modulating intrinsically photosensitive retinal ganglion cells. J. Neurosci. 2021, 41, 2566-2580. [CrossRef]

22. Murthy, K.R.; Goel, R.; Subbannayya, Y.; Jacob, H.K.; Murthy, P.R.; Manda, S.S.; Patil, A.H.; Sharma, R.; Sahasrabuddhe, N.A.; Parashar, A.; et al. Proteomic analysis of human vitreous humor. Clin. Proteom. 2014, 11, 29. [CrossRef] [PubMed]

23. Walker, F.; Patrick, R.S. Constituent monosaccharides and hexosamine concentration of normal human vitreous humour. Exp. Eye Res. 1967, 6, 227-232. [CrossRef]

24. Bårdsen, K.; Gjerstad, M.D.; Partinen, M.; Kvivik, I.; Tjensvoll, A.B.; Ruoff, P.; Omdal, R.; Brede, C. Considerably lower levels of hypocretin-1 in cerebrospinal fluid is revealed by a novel mass spectrometry method compared with standard radioimmunoassay. Anal. Chem. 2019, 91, 9323-9329. [CrossRef] [PubMed]

25. Hirtz, C.; Vialaret, J.; Gabelle, A.; Nowak, N.; Dauvilliers, Y.; Lehmann, S. From radioimmunoassay to mass spectrometry: A new method to quantify orexin-A (hypocretin-1) in cerebrospinal fluid. Sci. Rep. 2016, 6, 25162. [CrossRef] [PubMed]

26. Zhou, Y.; Wong, J.-M.T.; Mabrouk, O.S.; Kennedy, R.T. Reducing adsorption to improve recovery and in vivo detection of neuropeptides by microdialysis with LC-MS. Anal. Chem. 2015, 87, 9802-9809. [CrossRef]

27. US-FDA. Bioanalytical Method Validation Guidance for Industry, U.S.; Department of Health and Human Services Food and Drug Administration, Center for Drug Evaluation and Research (CDER) and Center for Veterinary Medicine (CVM), 7500 Standish Place; FDA: Rockvil, MD, USA, 2018.

28. Chrobok, L.; Jeczmien-Lazur, J.S.; Pradel, K.; Klich, J.D.; Bubka, M.; Wojcik, M.; Kepczynski, M.; Lewandowski, M.H. Circadian actions of orexins on the retinorecipient lateral geniculate complex in rat. J. Physiol. 2021, 599, 231-252. [CrossRef]

29. Chrobok, L.; Palus-Chramiec, K.; Chrzanowska, A.; Kepczynski, M.; Lewandowski, M.H. Multiple excitatory actions of orexins upon thalamo-cortical neurons in dorsal lateral geniculate nucleus-implications for vision modulation by arousal. Sci. Rep. 2017, 7, 7713. [CrossRef] 